\title{
Uncontrolled Systemic Inflammatory Response Syndrome by Cardiopulmonary Bypass F Sabzi $^{1}$, R Faraji $^{1}$
}

\begin{abstract}
It is known that the use of a cardiopulmonary bypass (CPB) during cardiac surgery leads to leukocyte activation and may, among other causes, induce organ dysfunction due to increased leukocyte recruitment into different organs. In our patients pathophysiologically severs SIRS, uncontrolled CPB induced inflammation, chylomicrons and very low density lipoproteins (VLDL) or immune complex have been shown to develop immune dependent agglutination by $\mathrm{C}$-reactive proteins in cardiopulmonary bypass, which could result in vascular occlusion and resultant infarction.
\end{abstract}

Keywords: Cardiopulmonary bypass, leukocyte, thoracic surgery

From: Preventive Cardiovascular Research Centre Kermanshah, Kermanshah University of Medical Sciences, Kermanshah, Iran.

Correspondence: R Faraji, Preventive Cardiovascular Research Centre Kermanshah, Kermanshah University of Medical Sciences, Kermanshah, Iran. Tel: +98 9183362603, Fax: +98 831 9360043. E-mail: r.faraji61@gmail.com 


\section{INTRODUCTION}

Socha found that during $\mathrm{CBP}$, phospholipase $\mathrm{A}_{2}$ degrades arachidonic acid, leading to inflammatory mediators such as leukotrienes, prostaglandins and thromboxanes. The action of these substances triggers adhesion and neutrophil activation, vasoconstriction, tissue injury, platelet aggregation and the ischaemic organ change (1). The differential diagnosis can be divided into three categories: emboli from the cardiac and arterial system, acquired hypercoagulability disorders and syndromes which lead to peripheral vascular pathology (2).

\section{CASE REPORT}

A 45 years-old woman secondary to rheumatic fever and aortic regurgitation was scheduled for AVR. Pre-operative echocardiography revealed severe aortic regurgitation. She denied any cerebral symptoms including headache, dizziness, transient ischaemic attacks or strokes on past medical history, the patient was found to be fully conscious, alert and oriented with stable vital signs. The blood pressure was $110 / 80 \mathrm{mmHg}$ and diastolic murmur was audible over mitral area. The physical exam revealed intact cranial nerves, motor power and sensation. There was no evidence of vasculitis such as Osler's nodes, Jane way pad, palmer erythema or cyanosis of fingers. The blood investigation showed an erythrocyte sedimentation rate $(\mathrm{ESR})$ of $4 \mathrm{~mm}$ with no leukocytosis $\left(\mathrm{WBC}=10.000 \mathrm{~mm}^{3}\right)$. The electrolytes and kidney and liver function were within normal limits. The chest X-ray was normal. Electrocardiogram (EKG) showed no change. The patient scheduled for $r e$ operative aortic valve replacement. Intra operatively, the aortic valve was tricuspid and was not calcified. The wall of the aorta in the sinus area appeared thin, but there was no root dilatation or wall calcification. A standard cardiopulmonary bypass (CPB) with bicaval cannulation was initiated, and cold cardioplegia was performed for myocardial protection after aortic cross-clamping. The aortic valve was 
carefully excised from the aortic annulus. After an appropriate prosthetic valve was selected, it was placed using five $2 / 0$ non absorbable monofilament polypropylene sutures with 1/2-circle 17-mm needles with continuous sutures. The aortotomy was closed with a doublelayer suture of 4-0 polypropylene the patient was weaned successfully off CPB with inotropic support and transferred to intensive cares unite. After six hours, blood pressure reduced and central venous pressure (CVP) elevated and extremity became cool and cyanotic. Inotropic support was started with, dobutamin and adrenaline ( 2 and $1 \mathrm{mic} / \mathrm{kg} / \mathrm{min}$ ) subsequently. After three days, inotropic drugs tapered and discontinued and blood pressure stabilized but cyanosis changed to dry gangrene and after two weeks acral part of lower and upper extremities auto amputated and specimen send to pathology. The possibility of systemic embolization was role out by echocardiography, and carotid Doppler study, and coagulation profile and a disseminated intravascular coagulation workup were all normal. The patient was started on aspirin, pentoxifylline and heparin. The parts were kept warm and undue handling was avoided. In histopathological exam, there was only non-specific vasulitis with thrombosis.

The patient has not regained fully consciousness on the following morning, however she was found to be quadriplegic with non-voluntary movements of the four limbs or face. Both pupils were small and fixed. Magnetic resonance imaging (MRI) of brain revealed normal great artery and edema of brain cortex. Prolonged ventilatory support was maintained and eventually a tracheostomy was performed to facilitate tracheobronchial suctioning and weaning. Facility for ultrasonography of the abdomen did not exist in our center; however abdominal ascitic fluid amylase was normal. A CT scan of the abdomen was not done as the patient could not be shifted. Acute renal failure with anemia managed and kidney recovered completely. However, the patient remained in coma state for four weeks with no neurological improvement. Despite all ventilatory, nutritional and nursing support, the patient died 
eventually of hepatic failure and generalized sepsis. Postoperative laboratories measurement includes negative antinuclear antibodies and rheumatoid factor, and elevation of $\mathrm{C} 4-\mathrm{C} 3$ and tissue necrotic factor alfa as components of complement, but HBS Antigen (especially in polyarteritis nodosa) was negative. The patient has not predisposing factors such as hypertension, arthrosclerosis or diabetes, and hypercoagubility state was role out by normal serum level of protein C-S, factor of $v$ leiden and von wilibr and disease. Pathologic exam showed no specific vasculitis as seen in inflammatory response in CPB.

\section{DISCUSSION}

Distal organ ischemia or acral parts involves arterioles with external diameters of 500-900 ウ̀m that may affected by variables pathology such as cholesterol emboli, immune complex produces by $\mathrm{CPB}$, atherosclerotic plaque or vasculitis syndrome (3). The most important differential diagnosis of CPB-induced SIRS is cholesterol crystal emboli (CCE).

Presence of four or more risk factors should be taken as presumptive evidence of CCE. These risk factors that were not found in our patients include atherosclerotic plaque, hypertension, thrombolitic therapy male sex, smoking, hypercholesterolaemia, diabetes and resuscitation (4). The pathognomic pathologic sign of CCE that was not seen in our patient was neddle shaped cleft in arteriole wall (5). Some studies found that however, various treatment strategies have been tested to reduce the severity of the systemic inflammation induced by $\mathrm{CPB}$ and to improve the treatment, including anti-inflammatory drugs, novel components of the CPB, and new surgical techniques, or anesthetic drugs or technique but no single strategy has been proven effective yet some of these drugs were evaluated in presiding studies (6-8). Production of humoral inflammatory mediators and priming of neutrophils by exposure to the CPB apparatus enables a 'post-pump' syndrome characterized by a systemic 
inflammatory response syndrome and its anti-inflammatory counterpart, termed the compensatory anti-inflammatory response syndrome (9) IL-18 plays a central role in regulating and balancing these responses. IL-18 regulates the expression of the potent proand anti-inflammatory mediators TNF- $\alpha$ (10) and IL-10 (11). In accord with this, Morgan found that the TT genotype was associated with an increased serum IL-18 concentration and also with increased serum TNF- $\alpha$ and decreased serum IL-10. The increased serum TNF- $\alpha$, and decreased serum IL-10 levels are associated with increased organ dysfunction (12).

\section{CONCLUSION}

The tremendous effect of the inflammatory response to ischaemia-reperfusion and the use of CPB indicate the need for measures that might if not inhibit it at least mitigate it. Thus, the control of risk factors, the reduction of ischaemic cardiovascular events, technical training for off-pump surgery, as well as advances in anti-inflammatory therapy, are measures to be reinforced while research should be encouraged so that these objectives are achieved. 


\section{REFERENCES}

1. Paparella D, Yau TM, Young E. Cardiopulmonary bypass induced inflammation: pathophysiology and treatment. Eur J Cardiothorac Surg 2002; 21: 232-244.

2. Socha LA, Gowardman J, Silva D, Correcha M, Petrosky N. Elevation in interleukin 13 levels in patients diagnosed with systemic inflammatory response syndrome. Intensive Care Med 2006; 32: 244-250.

3. Ben-Abraham R, Weinbroum AA, Dekel B, Paret G. Chemokines and the inflammatory response following cardiopulmonary bypass-a new target for therapeutic intervention. Paediatric Anaes 2003; 13: 651-665.

4. Applebaum RM, Kronzon I. Evaluation and management of cholesterol embolization and the blue toe syndrome. Curr Opin Cardiol 1996; 11: 533-42.

5. Halter J, Steinberg J, Fink G, Lutz C, Picone A, Maybury R, et al. Evidence of systemic cytokine release in patients undergoing cardiopulmonary bypass. J Extra Corpor Technol 2005; 37: 272-277.

6. Braude S, Nolop KB, Fleming JS, Krausz T, Taylor KM, Royston D. Increased pulmonary transvascular protein flux after canine cardiopulmonary bypass. Association with lung neutrophil sequestration and tissue peroxidation. Am Rev Res Dis 1986; 134: 867-872.

7. Abdel-Rahman U, Margraf S, Aybek T, Logters T, Bitu-Moreno J, Francischetti I, et al. Inhibition of neutrophil activity improves cardiac function after cardiopulmonary bypass. J inflammation 2007; 4: 21.

8. Baki ED, Aldemir M, Kokulu S, Koca HB, Ela Y, Sivacı RG, et al. Comparison of the effects of desflurane and propofol anesthesia on the inflammatory response and s100 $\beta$ protein during coronary artery bypass grafting. Inflammation 2013; 36: 1327-33. 
9. Spriggs DR, Sherman ML, Frei E, Kufe DW. Clinical studies with tumour necrosis factor. Ciba Found Symp 1987; 131: 206-227.

10. Ley K, Laudanna C, Cybulsky MI, Nourshargh S. Getting to the site of inflammation: the leukocyte adhesion cascade updated. Nat Rev Immunol 2007; 7: 678-689.

11. Cameron D. Initiation of white cell activation during cardiopulmonary bypass: cytokines and receptors. J Cardiovasc Pharmacol 1996; 1: 1-5.

12. Pinsky MR, Vincent JL, Deviere J, Alegre M, Kahn RJ, Dupont E. Serum cytokine levels in human septic shock. Relation to multiple-system organ failure and mortality. Chest 1993; 103: 565-575. 


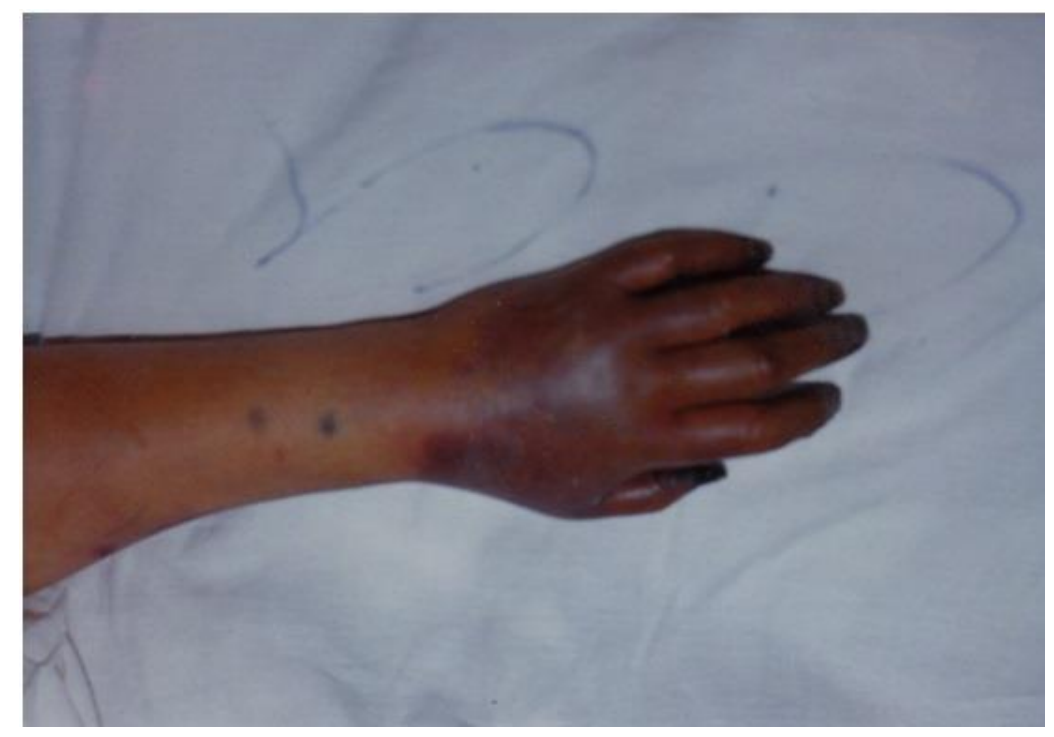

Fig 1. Shows left hand ischaemia.

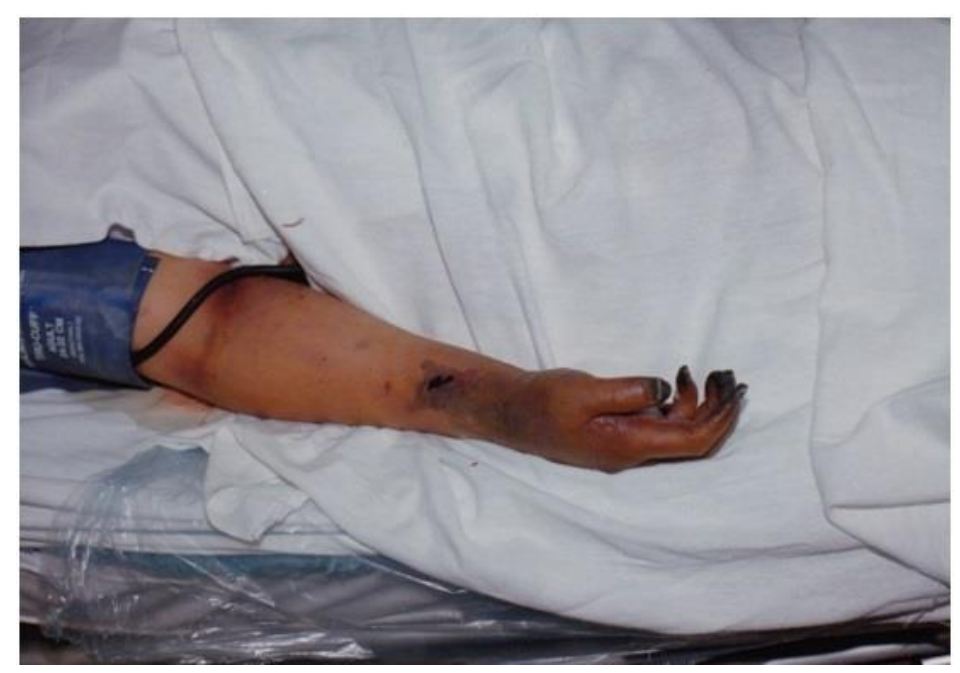

Fig 2. Shows right hand ischaemia.

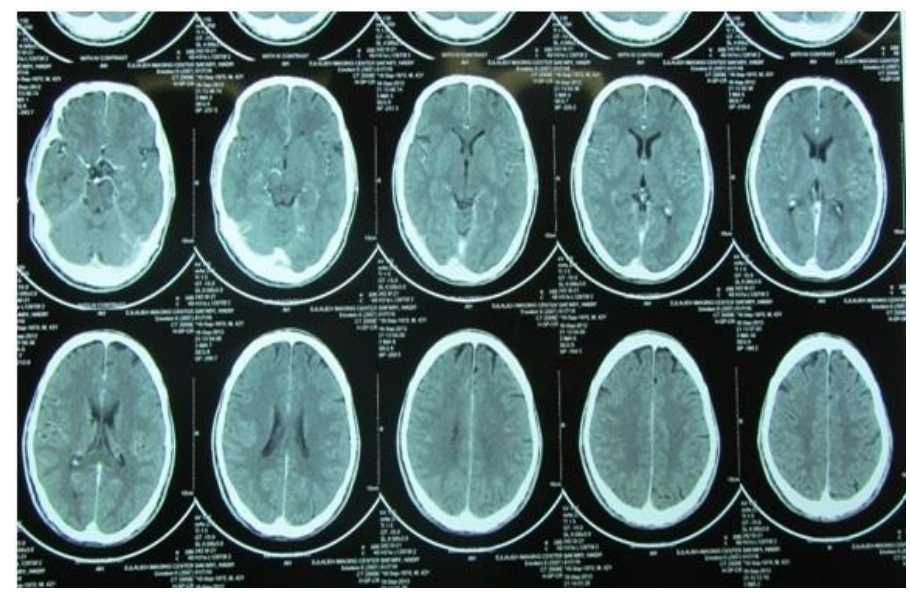

Fig 3. Shows non-specific brain edema caused by inflammation. 